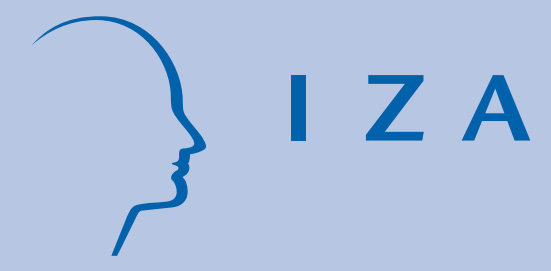

IZA Standpunkte Nr. 47

Ökonomische Ursachen und Folgen von Migration

Klaus F. Zimmermann

Januar 2012

Forschungsinstitut zur Zukunft der Arbeit Institute for the Study of Labor 


\title{
Ökonomische Ursachen und Folgen von Migration
}

\author{
Klaus F. Zimmermann
}

IZA und Universität Bonn

IZA Standpunkte Nr. 47

Januar 2012

IZA

Postfach 7240

53072 Bonn

Tel.: (0228) 3894-0

Fax: (0228) 3894-180

E-Mail: iza@iza.org

Die Schriftenreihe "IZA Standpunkte" veröffentlicht politikrelevante Forschungsarbeiten und Diskussionsbeiträge von IZA-Wissenschaftlern, IZA Research Fellows und IZA Research Affiliates in deutscher Sprache. Die Autoren sind für den Inhalt der publizierten Arbeiten verantwortlich. Im Interesse einer einheitlichen Textzirkulation werden Aktualisierungen einmal publizierter Arbeiten nicht an dieser Stelle vorgenommen, sondern sind gegebenenfalls nur über die Autoren selbst erhältlich. 
IZA Standpunkte Nr. 47

Januar 2012

\section{ZUSAMMENFASSUNG}

\section{Ökonomische Ursachen und Folgen von Migration*}

Der Beitrag gibt einen Einblick in das ökonomische Verständnis der Ursachen und Folgen wirtschaftlich bedingter Migration. In ökonomischen Systemen ist Arbeitskräftemobilität für die Verbesserung der Allokation und der Verteilung der volkswirtschaftlichen Ressourcen sehr wesentlich. Angesichts eines wachsenden Fachkräftemangels bei weiter hoher Arbeitslosigkeit unter gering Qualifizierten bleibt die Anwerbung internationaler und europäischer Fachkräfte eine zentrale politische Gestaltungsaufgabe für jede Volkswirtschaft. Der Beitrag schließt deshalb mit einer Bewertung der neuesten Initiativen zur Steuerung qualifizierter Zuwanderung in Deutschland und Österreich.

JEL-Codes: F22, J21, J61

Schlagworte: Zuwanderungspolitik, Arbeitsmigration, Integration, Ungleichheit, Punktesystem, Demografischer Wandel

Kontaktadresse:

Klaus F. Zimmermann

IZA

Postfach 7240

D-53072 Bonn

E-Mail: direktor@iza.org

\footnotetext{
* Dies ist eine überarbeitete Fassung meines Vortrages beim Österreichischen Wissenschaftstag 2011 zum Thema Migration in Semmering. Ich bin Ulf Rinne und Teilnehmern der Tagung für Anregungen und Hinweise sehr dankbar. Diese Darlegungen stützen sich auf vielfältige eigene Aufarbeitungen zum Thema, bsw. Holger Hinte, Ulf Rinne und Klaus F. Zimmermann (2011): Zuwanderung, Demografie und Arbeitsmarkt: Fakten statt Vorbehalte, IZA Standpunkte Nr. 37, und Amelie F. Constant und Klaus F. Zimmermann, Migration, Ethnicity and Economic Integration, in: Miroslav N. Jovanovic (ed.), International Handbook of Economic Integration. Edward Elgar Publishing, Cheltenham, 2011, 145168.
} 


\section{Einleitung}

Der vorliegende Beitrag schlägt einen Bogen von der Diskussion der ökonomischen Dimension von Zuwanderung und Integration bis hin zu den neuerdings in Österreich wie auch in Deutschland intensivierten Debatte um eine aktive Steuerung qualifizierter Zuwanderung. Es werden die ökonomischen Ursachen und Folgen von Migration anhand der Ergebnisse einer Reihe von Studien diskutiert und so die grundlegenden ökonomischen Zusammenhänge transparent und verständlich gemacht. ${ }^{1}$ Ein solches Verständnis ist wichtig - nicht nur für die Formulierung von Politikmodellen, wie sie zuletzt Österreich mit der „Rot-Weiß-Roten Karte“ vorgenommen hat, sondern auch für deren Vermittlung in der Öffentlichkeit. Denn der Erfolg von Migrationspolitik bemisst sich auch am Grad der Zustimmung der einheimischen Bevölkerung zu etwaigen Politikmaßnahmen.

Arbeitskräftemobilität ist zentral für die Verbesserung der Allokation und die Verteilung volkswirtschaftlicher Ressourcen. Angesichts eines wachsenden Fachkräftemangels bei weiter hoher Arbeitslosigkeit unter gering Qualifizierten wird die Anwerbung internationaler und europäischer Fachkräfte zu einer wichtigen politischen Gestaltungsaufgabe. Zusätzliche Fachkräfte bringen Wohlstand und bauen wirtschaftliche Ungleichheit ab, da sie auch gering Qualifizierte in Arbeit bringen. Die globalisierte Welt und die demografischen Verwerfungen verlangen nach einer schnellen Anpassung. Mit der europäischen Integration und insbesondere dem gemeinsamen EuroWährungsraum sind zudem Wechselkurse und Außenwirtschaftspolitik als

\footnotetext{
${ }^{1}$ Vgl. für eine Einführung in die ökonomische Analyse den Handbuchartikel von Amelie F. Constant und Klaus F. Zimmermann, Migration, Ethnicity and Economic Integration, in: Miroslav N. Jovanovic (ed.), International Handbook of Economic Integration. Edward Elgar Publishing, Cheltenham, 2011, 145 - 168. Dieser Survey referiert über den Kernbestand der Migrationsökonomie auch die ökonomischen Ansätze zur ethnischen Identitätsforschung, die im Beitrag von Jean Phinney, Acculturation of migrants: The contribution of the receiving society, in diesem Tagungsband, angesprochen werden.
} 
Flexibilitätsinstrumente entfallen. Auch deswegen hat sich die Bedeutung flexibler Arbeitsmärkte verstärkt. In dem Maße wie Europa zusammenwächst müssen sie für einen größeren Teil der nötigen Anpassungsreaktionen sorgen.

Vor diesem Hintergrund sind politische Initiativen zur Stärkung der ökonomischen Zuwanderungskomponente von erheblicher Bedeutung. Der vorliegende Beitrag argumentiert zunächst auf Basis der deutschen Erfahrungen. Er skizziert einen Vorschlag für ein Punkte-Auswahlsystem für die Zuwanderung von Arbeitskräften nach Deutschland. Abschließend folgt in diesem Zusammenhang eine erste Bewertung der österreichischen „Rot-WeißRoten Karte“.

\section{Zuwanderung in ökonomischer Betrachtung}

Ökonomische Modelle unterstellen typischerweise, dass die Entscheidung zur Migration in einem rationalen Kalkül unter Berücksichtigung des zu erwartenden Nutzens sowie der entstehenden monetären und psychischen Kosten (etwa durch Umzug und Trennung von Familie oder sozialem Umfeld) getroffen wird. In diesem Rahmen vermögen Analysen der weltweiten Wanderungsbewegungen die wesentlichen Beweggründe der Entscheidung zur Migration und ihre Auswirkungen identifizieren. Auf diese Weise stellt die Migrationsforschung den politischen Entscheidungsträgern eine Grundlage bereit, auf der sich evidenzbasierte Politikkonzepte entwickeln lassen.

Eine rein ökonomische Betrachtung von Zuwanderung ist jedoch vielen Menschen suspekt. Diese Sichtweise ignoriere die humanitäre Dimension und reduziere Zuwanderer auf ihren Nutzen als Arbeitskraft. Die Forderung nach einer expliziten „Auswahl“ von Zuwanderern und klaren Integrationsanforderungen erscheint in dieser Perspektive zumindest anrüchig.

Dabei wird jedoch ausgeblendet, dass es gute Gründe für eine Begrenzung von Zuwanderung geben kann, die man sorgfältig und transparent 
abwägen muss. Eine Analyse der Faktenlage verdeutlicht, dass die Zuwanderung qualifizierter Arbeitskräfte generell klare ökonomischen Vorteile bringt. ${ }^{2}$ Obwohl etwa in Deutschland Zuwanderung bislang eher passiv hingenommen denn aktiv gestaltet wurde, fällt die Bilanz der jüngeren Zuwanderungsgeschichte sogar unter diesen Voraussetzungen per Saldo günstig aus. Eine aktive und steuernde Zuwanderungspolitik wird die Wohlfahrtsgewinne durch Zuwanderung weiter vergrößern und ist ein wichtiges Fundament für eine noch größere Akzeptanz von Migration in unserer Gesellschaft.

\section{Zuwanderung schafft Arbeitsplätze und reduziert Ungleichheit}

Hinsichtlich der Zuwanderung gibt es derzeit noch viele Vorbehalte. Häufig wird die Befürchtung geäußert, dass die Beschäftigung von Einheimischen durch Zuwanderung gefährdet ist. Die simplifizierende Rechnung, dass ein zusätzlich erwerbstätiger Zuwanderer unweigerlich $\mathrm{zu}$ einem arbeitslosen Einheimischen führt, ist jedoch falsch. Denn ein zusätzlicher beschäftigter qualifizierter Zuwanderer erzeugt neue Nachfrage nach geringer qualifizierter Arbeit - etwa im Bereich einfacher Dienstleistungen. Im Ergebnis entstehen so im Umfeld jeder erwerbstätigen Fachkraft bis zu drei zusätzliche Arbeitsplätze für Geringqualifizierte.

Die Zuwanderung von Fachkräften führt also zu einer steigenden Nachfrage nach geringer qualifizierter Arbeit, was wiederum auch einen Anstieg des Lohnniveaus in diesem Segment nach sich zieht. Berechnungen für die USA zeigen, dass rund 90 Prozent der Einheimischen einen Lohnanstieg aufgrund von Zuwanderung verzeichnen konnten. ${ }^{3}$ Damit führt

\footnotetext{
${ }^{2}$ Vgl. Holger Hinte, Ulf Rinne und Klaus F. Zimmermann (2011): Zuwanderung, Demografie und Arbeitsmarkt: Fakten statt Vorbehalte, IZA Standpunkte Nr. 37.

${ }^{3}$ Vgl. Gianmarco Ottaviano und Giovanni Peri (2006): The Economic Value of Cultural Diversity: Evidence from US Cities, Journal of Economic Geography, 6(1), S. 9-44.
} 
Zuwanderung zu positiven Lohneffekten für den überwiegenden Teil der Bevölkerung.

Allerdings steigen gleichzeitig die Löhne der qualifizierten Einheimischen bei Zuwanderung von Fachkräften nicht in dem Maße, wie sie bei Fachkräftemangel sonst gestiegen wären. Die relative Einkommensposition der besser Verdienenden verschlechtert sich somit und die ungleiche Verteilung der Einkommen wird geringer. Dieser Effekt lässt sich mit einer empirischen Untersuchung der Einkommensverteilung in Westeuropa belegen. ${ }^{4}$ Hier zeigte sich, dass die Ungleichheit der Einkommen mit steigendem Bevölkerungsanteil der Ausländer abnahm. ${ }^{5}$

Die genannten ökonomischen Zusammenhänge der Zuwanderung in Bezug auf die Lohnentwicklung widerlegen somit die verbreitete Meinung, dass Zuwanderung sozialen Sprengstoff beinhalten muss. Das Gegenteil ist zutreffend: Wenn keine Fachkräfte ins Inland geholt werden, verschlechtert das die Chancen geringer qualifizierter Menschen weiter. Es entsteht dann erst recht eine Zwei-Klassen-Gesellschaft, in der die einen kaum Chancen auf dem Arbeitsmarkt haben und die anderen so stark gesucht sind, dass sie maximale Einkommen und Privilegien einfordern können. Hierin liegt eine erhebliche Brisanz und eine derartige Entwicklung beinhaltet tatsächlich sozialen Sprengstoff.

\footnotetext{
${ }^{4}$ Vgl. Klaus F. Zimmermann und Martin Kahanec (2009): International Migration, Ethnicity and Economic Inequality, in: Wiemer Salverda, Brian Nolan und Timothy M. Smeeding (Hrsg.): The Oxford Handbook of Economic Inequality, Oxford, S. 455-490.

${ }^{5}$ Wanderungsbewegungen bestimmen auch sehr langfristig die Verteilung des weltweiten Wohlstandes. Der Beitrag von Johannes Krause, Der Urmensch in uns: Genetische Vermischungen bei der Ausbreitung des modernen Menschen, in diesem Tagungsband, dokumentiert die Verbreitung und Vermischung des genetischen Codes. Die Studie von Quamrul Ashraf und Oded Galor, The Out of Africa Hypothesis, Human Genetic Diversity and Comparative Economic Development, die im American Economic Review erscheinen wird, zeigt, dass etwa $15 \%$ der gegenwärtigen globalen Variation der Pro-KopfEinkommensverteilung durch Faktoren erklärbar sind, die über 100000 Jahre zurückliegen und mit den seinerzeitigen Wanderungsbewegungen zusammenhängen.
} 


\section{Zuwanderung entlastet die öffentlichen Haushalte}

Eine weitere populäre These, die häufig gegen Zuwanderung vorgebracht wird, besagt, dass die hohe Arbeitslosigkeit von Ausländern für eine erhebliche Kostenbelastung der öffentlichen Haushalte sorge. Zuwanderung führe so de facto zum Einfall in die sozialen Sicherungssysteme.

Empirische Befunde widersprechen diesem Bild jedoch entschieden. So haben im Jahr 2004 die aus Steuern und Sozialabgaben von Ausländern resultierenden deutschen Staatseinnahmen die Transferausgaben an Ausländer mit rund 2.000 Euro pro Person deutlich überschritten. ${ }^{6}$ Die Hauptursache dafür ist die vergleichsweise günstige Alterstruktur dieser Bevölkerungsgruppe. Gleichzeitig verdeutlicht die Analyse, dass sich durch eine bessere Arbeitsmarktintegration die Bilanz der Ausländer noch deutlich steigern ließe.

Obwohl Untersuchungen über die Inanspruchnahme der Leistungen des Wohlfahrtsstaates gezeigt haben, dass die Inanspruchnahme der Zuwanderer über derjenigen der Einheimischen liegt, findet auch das Bild des „Wohlfahrtstourismus“ keine Bestätigung und ist bei näherer Betrachtung in ganz Europa unbegründet. ${ }^{7}$ So ist zwar beispielsweise in Deutschland das Arbeitslosigkeitsrisiko unter Ausländern etwa doppelt so hoch wie unter Einheimischen. Berücksichtigt man in dieser Betrachtung jedoch Faktoren wie etwa Alter, Geschlecht, Ausbildung, Art der Beschäftigung und Berufserfahrung, so stellt man fest, dass die Rate der Inanspruchnahme von Sozialleistung dann in der einheimischen Bevölkerung höher ist als unter den vergleichbaren Ausländern.

\footnotetext{
${ }^{6}$ Vgl. Holger Bonin (2006): Der Finanzierungsbeitrag der Ausländer zu den deutschen Staatsfinanzen: Eine Bilanz für 2004, IZA Discussion Paper, Nr. 2444.

${ }^{7}$ Vgl. Corrado Giulietti, Martin Guzi, Martin Kahanec und Klaus F. Zimmermann (2011): Unemployment Benefits and Immigration: Evidence from the EU, IZA Discussion Paper, Nr. 6075 .
} 
Diese Ergebnisse sprechen dafür, dass der höhere Anteil von Arbeitslosen unter Zuwanderern mit einer falschen Migrations- und Integrationspolitik und einer fehlenden Zuwanderungssteuerung zusammenhängt.

\section{Die verpasste Chance der EU-Osterweiterung}

Die oben genannten Effekte der Zuwanderung lassen sich anhand der Erfahrungen im Zuge der EU-Osterweiterung in den Jahren 2004 und 2007 verdeutlichen. ${ }^{8}$ Es zeigt sich, dass sich die verspätete Öffnung der Arbeitsmärkte in Deutschland und Österreich für diese beiden Länder nachteilig ausgewirkt hat. Andere Länder (vor allem Irland, Schweden und Großbritannien) haben im Gegenzug davon profitiert, schon frühzeitig die Freizügigkeit für die neuen EU-Bürger aus Osteuropa realisiert zu haben.

Die Gesamtzahl der Zuwanderer aus den neuen Mitgliedstaaten hat zwischen 2003 und 2007 bei sehr ungleicher Verteilung zwischen den Zielländern der alten EU deutlich zugenommen. Von einer Massenmigration, wie sie vor der EU-Osterweiterung befürchtet worden war, kann allerdings keine Rede sein. Der Anteil der Einwanderer aus den Beitrittsländern in die alten EU-Staaten ist weiterhin geringer als derjenige von Zuwanderern aus Drittstaaten. Die praktizierten Übergangsregelungen zur Einschränkung der Arbeitnehmerfreizügigkeit haben offenkundig viele qualifizierte Zuwanderer aus den Beitrittsländern des Jahres 2004 nach Irland oder Großbritannien „umgeleitet“, während Spanien und Italien die primären Zielländer für Einwanderer aus Bulgarien und Rumänien sind.

\footnotetext{
${ }^{8}$ Vgl. Ulf Rinne und Klaus F. Zimmermann (2009): Fünf Jahre EU-Ostererweiterung: Schlechte Noten für deutsche Abschottungspolitik, IZA Standpunkte Nr. 14, und Martin Kahanec und Klaus F. Zimmermann (2009): EU Labor Markets after Post-Enlargement Migration, Springer-Verlag, Berlin et. al.
} 
Die Bilanz der EU-Osterweiterung zeigt darüber hinaus, dass Zuwanderer keineswegs häufiger sozialstaatliche Leistungen in Anspruch genommen haben als Einheimische. Es gibt auch keine Belege für einen Rückgang des Lohnniveaus oder eine Verdrängung einheimischer Beschäftigung. Stattdessen gibt es Hinweise auf einen Rückgang der Ungleichheit bei den Einkommen in den alten EU-Staaten. Obwohl der „Brain Drain“ - also die Abwanderung von qualifizierten Arbeitskräften - in den Herkunftsländern durchaus spürbar ist, sprechen die bisherigen Analysen dafür, dass die Fachkräftezirkulation innerhalb der EU insgesamt zu einer besseren Lösung der demographischen und ökonomischen Probleme beitragen und die Allokationseffizienz in der EU verbessern kann. So dürfte die zu erwartende „Wissenszirkulation“ im Rahmen von zirkulärer Migration und Rückwanderung einen Beitrag leisten, die demografische und ökonomische Herausforderung der Abwanderung von jungen und qualifizierten Personen in den Herkunftsländern zu bewältigen.

Im Zuge der EU-Osterweiterung haben sich also viele wanderungswillige qualifizierte Osteuropäer für andere Länder entschieden, während sie in Deutschland und Österreich weiterhin fehlen. Beide Länder haben durch die Verzögerungstaktik bei der Öffnung ihrer Arbeitsmärkte eine Chance verpasst.

\section{Determinanten von Zuwanderung}

Ökonomischen Modellen zufolge reagiert Arbeitsmigration stark auf objektive wie subjektiv empfundene Unterschiede in der regionalen Wohlfahrt, vor allem in Form von Lohngefällen und unterschiedlichen Arbeitslosigkeitsraten. Die Wanderungsentscheidung wird dabei in einem Kalkül unter Berücksichtigung verschiedener Faktoren getroffen, wie beispielsweise die 
entstehenden monetären und psychischen Kosten durch Umzug und Trennung von Familie oder dem sozialen Umfeld. ${ }^{9}$

So wandern nach dem Humankapitalmodell ältere Menschen mit geringerer Wahrscheinlichkeit, denn der erwartete Nutzen über den Lebenszyklus aus der Wanderung ist für sie geringer als für jüngere Menschen. Individuen mit besserer Ausbildung neigen in stärkerem Maße zur Migration, da sie nicht nur höhere Einkommen erwarten können, sondern auch die Risiken der Wanderung besser abschätzen können. Die Risiken und Kosten der Wanderung wachsen typischerweise mit der Entfernung zum Zielort, auch weil die Informationen über entfernte Arbeitsmärkte schwieriger zu erhalten sind. Die Entscheidung zur Migration vollzieht sich ferner im Kontext familiärer und ethnischer Netzwerke.

Wenn weiterhin eine mangelnde Beweglichkeit der Arbeitsmärkte auf der nationalen wie der europäischen Ebene beklagt wird, so hat dies eine Fülle von Ursachen. So macht die zunehmende Erwerbsbeteiligung von Frauen und damit die einhergehende Zunahme von Doppelverdienerhaushalten die Mobilitätsentscheidung komplexer. Eine Wanderung wird im Ergebnis weniger wahrscheinlich. Weitere Ursachen für die anhaltend schwache Arbeitskräftemobilität könnten unter anderem in der Zunahme von Wohneigentum, der begrenzten Übertragbarkeit von Ansprüchen an soziale Sicherungssysteme und der fehlenden Anerkennung von formalen Qualifikationen liegen. Eine verbesserte Verkehrinfrastruktur mit schnelleren Zügen und preiswerteren Flügen hat ambivalente Wirkungen. Sie kann zwar Arbeitsplatzwechsel in entfernte Regionen stimulieren, aber auch das Pendeln erleichtern und überregionale Präsenz ohne einen solchen Wechsel begünstigen. Schließlich sind weiterhin mangelnde Sprachkenntnisse und

\footnotetext{
9 Vgl. Klaus F. Zimmermann (2008): Mobilitätspolitik in Europa, Wirtschaftspolitische Blätter, 2, S. 199-208.
} 
kulturelle Unterschiede die wirksamsten Hemmnisse für die geographische Mobilität, nicht nur innerhalb Europas.

Trotz einer weiterhin geringen Mobilität ist insgesamt die Bereitschaft zur Mobilität heute größer als noch vor einigen Jahren. Zudem vollziehen sich Wanderungsbewegungen über größere räumliche Distanzen. Während früher das Bild des Gastarbeiters prägend war, der bleibt, so verstehen wir heute Wanderungsströme als einen komplexeren Prozess. Zirkuläre Migration und Kettenmigration gewinnen an Bedeutung. Bei der Kettenmigration vollzieht sich die Wanderung in Netzwerken familiärer, sozialer oder ethnischer Gruppen, in der Individuen einander folgen. Zirkuläre Migration erfasst die Tendenz, nicht zu bleiben, sondern insbesondere durch die Bedingungen der Arbeitsmärkte getrieben weiter zu ziehen, ins Ursprungsland zurückzukehren und auch wieder in das ursprünglich gewählte Zielland zurückzukehren.

Politische Entscheidungsträger müssen folglich damit rechnen, dass Politikmaßnahmen, die Migration unterbinden wollen, das Gegenteil ihres intendierten Effektes bewirken, wenn sie auf zirkuläre Migration und Kettenmigration stossen. ${ }^{10}$ Hürden, physische oder juristische Mauern gegenüber Immigration behindern dann die natürliche Emigration insbesondere von Arbeitsmigranten und verursachen einen Nachzug familärer und ethnisch verbundener Gruppen.

Eine moderne Zuwanderungspolitik muss deshalb die nötigen Freiräume für Weiter- und Rückwanderung schaffen. Dies gilt umso mehr, als sich gerade qualifizierte Zuwanderer sonst kaum für das eigene Land gewinnen lassen.

\footnotetext{
${ }^{10}$ Vgl. Amelie F. Constant und Klaus F. Zimmermann (2011): Circular and Repeat Migration: Counts of Exits and Years Away from the Host Country, Population Research and Policy Review, 30 (4), S. 495-515.
} 


\section{Demografischer Wandel und Fachkräftemangel}

Zwar herrscht derzeit noch kein allgemeiner und flächendeckender Fachkräftemangel in Deutschland, aber es zeigen sich erste Engpässe in bestimmten Berufen, für bestimmte Qualifikationsniveaus, sowie in einzelnen Regionen und Branchen. ${ }^{11}$ Beispiele dafür sind Ingenieurberufe (Maschinen-, Fahrzeugbau- und Elektroingenieure) und bestimmte Gesundheitsberufe.

Hinzu kommt, dass sich das Fachkräfteproblem durch den demografischen Wandel in Zukunft deutlich verschärfen wird. Bis zum Jahr 2020 wird die Schülerzahl voraussichtlich um mehr als 10 Prozent abnehmen, wobei gerade in Ostdeutschland viele Regionen besonders betroffen sind. Immer mehr Ausbildungsplätze bleiben bereits heute unbesetzt. Das Arbeitskräftepotenzial wird bis 2025 um 6,5 Millionen Menschen sinken und bereits ab dem Jahr 2013 verliert Deutschland alle drei Jahre rund eine Million Personen im erwerbsfähigen Alter. Während aktuell in Deutschland noch knapp 54 Millionen Einwohner im Alter zwischen 15 und 64 Jahren leben, wird diese Zahl selbst unter optimistischen Annahmen bis zum Jahr 2050 auf 42 Millionen sinken.

Als Folge könnte ein Fachkräftemangel so das Wirtschaftswachstum in Deutschland schon bald verlangsamen. Die fehlenden qualifizierten Kräfte verursachen aber auch einen Ausfall an Nachfrage nach einfacher Arbeit, die ohnedies durch technischen Fortschritt sinkt. Arbeitslosigkeit im Niedrigqualifikationsbereich ist die schmerzliche Folge.

\footnotetext{
11 Der sich abzeichnende Fachkräftemangel in Deutschland ist immer wieder öffentlich thematisiert worden, so bsw. vom Chef der Bundesagentur für Arbeit, Frank-Jürgen Weise, s. Kölner Stadtanzeiger vom 11. 1. 2012 ("Millionen Arbeitskräfte fehlen"). Siehe auch Gerd Zika und Robert Helmrich (2011), Qualifikations- und Berufshauptfeldprojektionen bis 2025: Fachkräftemangel: Es sind nicht nur die MINT-Berufe betroffen, Sozialer Fortschritt, 60 (8), 161-168. Dies gilt auch in Österreich und der Schweiz.
} 


\section{Politische Handlungsoptionen}

Eine Linderung des Fachkräftemangels kann durch eine stärkere Aktivierung inländischer Potenziale erfolgen und die Politik kann dies durch Massnahmen unterstützen. Dies betrifft etwa die Weiterbeschäftigung und Weiterqualifizierung Älterer. Reformen der Frühverrentung haben bereits eine verstärkte Weiterbeschäftigung Älterer bewirkt. Es bestehen jedoch auch hinsichtlich älterer Arbeitsloser sowie bezüglich der Verankerung von „lebenslangem Lernen“ in der Gesellschaft weitere Möglichkeiten zur Steigerung des Arbeitskräftepotenzials. ${ }^{12}$

Darüber hinaus wird argumentiert, dass die Frauenerwerbsquote durch eine familienfreundliche Infrastruktur in Betrieben und der Gesellschaft weiter erhöht werden könne. Auf diese Weise könnten bis zu 1,5 Millionen Vollzeitstellen durch aktuell nicht erwerbstätige Mütter besetzt werden - wenn diese die Möglichkeit hätten, ihre Arbeitszeit flexibler zu gestalten. ${ }^{13}$ Weitere inländische Potenziale können durch eine bessere Integration von Personen mit Migrationshintergrund, eine Verlängerung der Lebensarbeitszeit sowie eine Ausweitung der Wochenarbeitszeit aktiviert werden. Eine andere Handlungsoption stellt die qualifizierte Zuwanderung dar.

\section{Ein Plädoyer für mehr Zuwanderung}

Die Demografie lässt Arbeitslosigkeit nicht „von selbst“ verschwinden. Im Gegenteil: Eine sich öffnende Schere zwischen verfestigter Arbeitslosigkeit von Geringqualifizierten und sehr guten Marktchancen von Fachkräften zeichnet sich als gesellschaftliche Perspektive ab.

\footnotetext{
${ }^{12}$ Vgl. Karl Brenke und Klaus F. Zimmermann (2011): Ältere auf dem Arbeitsmarkt, DIWVierteljahreshefte 80 (2), 11-31, und Werner Eichhorst (2011): The Transition from Work to Retirement, IZA Discussion Paper Nr. 5490.

${ }^{13}$ Vgl. Werner Eichhorst, Paul Marx und Verena Tobsch (2010): Familienfreundliche flexible Arbeitszeiten - ein Baustein zur Bewältigung des Fachkräftemangels, IZA Research Report Nr. 33.
} 
Eine gezielte Zuwanderung von Fachkräften erscheint deshalb unverzichtbar. Sie bietet einerseits einen flexiblen „Flankenschutz“ für andere Politikmaßnahmen und andererseits bewegt sich der künftige Arbeitskräftebedarf weit jenseits dessen, was sich ohne Zuwanderung auffangen lassen wird. Schließlich entfalten die oben genannten Maßnahmen zur Aktivierung inländischer Potenziale ihre Wirkung nur mit erheblicher zeitlicher Verzögerung.

Hinsichtlich der Zuwanderungs- und Integrationspolitik sind aktuell in Deutschland jedoch deutliche Defizite zu erkennen. Im Ergebnis ist der Saldo von Zuwanderung und Abwanderung seit Jahren nahezu ausgeglichen (und in einigen Jahren sogar negativ). Im Jahr 2011 kam es allerdings wieder zu einem erheblichen Zustrom nach Deutschland. Dabei war die gute Verfassung des deutschen Arbeitsmarktes und die kritische Lage der Wirtschaft in den Südländern der Europäischen Union ausschlaggebend. ${ }^{14}$

Die Hintergründe für die Politikmängel sind vielschichtig. So findet Zuwanderung weitgehend ungesteuert statt, Bildungs- und Berufsabschlüsse werden weiterhin zu oft nicht anerkannt, es fehlt eine kritische Evaluation der Effektivität und Effizienz der Integrationspolitik und das Potenzial ausländischer Studienabsolventen wird nicht ausreichend genutzt.

Auch in der europäischen Perspektive ist Deutschland eher Prellbock und nicht Lokomotive in der Migrationspolitik. Dabei ist Europa in Bewegung: Neben den klassischen Einwanderungsländern wie etwa Kanada und Australien, die auf ein Punktesystem setzen, finden auch in Europa Zuwanderungsgesetze zunehmend Verbreitung, so beispielsweise in Großbritannien, Dänemark und Österreich. Dadurch gerät Deutschland zusehends in die Defensive.

\footnotetext{
${ }^{14}$ Der deutsche Wirtschaftsminister wie die Bundesagentur für Arbeit haben zu Recht die Beschäftigung Arbeitsloser aus diesen Staaten gefördert. Die daran geäußerte öffentliche Kritik geht an der ökonomischen und politischen Rationalität vorbei.
} 
Dabei ist eine entschlossene Reaktion auf den demografischen Wandel dringend erforderlich. Allerdings entfalten Anpassungen der Renten- und Familienpolitik, altersgerechte Arbeit und Entlohnung ihre Effekte jedoch erst mit zeitlicher Verzögerung. Insofern ist eine Anpassung der Zuwanderungspolitik geboten. Hier ist es wichtig sowohl eine temporäre als auch eine permanente Komponente zu etablieren, um auf den kurzfristigen und langfristigen Bedarf reagieren zu können.

Je offensiver und glaubwürdiger Deutschland um „kluge Köpfe“ wirbt, umso eher treten bereits kurzfristig positive Wirkungen ein. Die Bundesregierung ist zum Handeln aufgefordert, aber auch Unternehmen müssen sich stärker engagieren, etwa durch gezielte Werbung um Fachkräfte in Osteuropa, in Nordafrika oder der Türkei.

\section{Ein kombiniertes Quoten- und Punktesystem für Deutschland}

Eine kombiniertes Quoten- und Punktesystem stellt eine attraktive Option zur bedarfsgerechten Steuerung von Zuwanderung dar. Es existiert auch bereits ein sehr konkreter Vorschlag, wie eine solches System in Deutschland aussehen könnte. ${ }^{15}$ Eine Umsetzung ist jedoch bislang nicht absehbar, obgleich sich der Vorschlag sehr pragmatisch in das geltende Zuwanderungsgesetz aufnehmen ließe.

Das Kernstück des umfassenden Gesamtkonzeptes bildet eine DreiSäulen-Strategie, die sowohl den Zuzug Hochqualifizierter, die Einwanderung qualifizierter Fachkräfte mit nachgefragten Qualifikationen als auch temporäre Arbeitsmigration zur Deckung eines kurzfristigen Zuwanderungsbedarfes aufgrund berufsspezifischer Nachfrageüberschüsse steuert.

\footnotetext{
${ }^{15}$ Vgl. Holger Hinte und Klaus F. Zimmermann (2010): Agenda Zuwanderung: Zehn-PunkteAktionsplan des IZA für gesteuerte Arbeitsmigration und bessere Integration, IZA Standpunkte Nr. 32; Holger Hinte Ulf Rinne und Klaus F. Zimmermann (2011): Ein Punktesystem zu bedarfsorientierten Steuerung der Zuwanderung nach Deutschland, IZA Research Report Nr. 35.
} 
Die erste Säule zielt auf die Steuerung der Zuwanderung Hochqualifizierter ab. Diese Gruppe verfügt über flexible und übertragbare Qualifikationen und Fähigkeiten, die vor dem Hintergrund einer sich schnell wandelnden Wissensgesellschaft zunehmend an Bedeutung gewinnen, aber gleichzeitig auch eine hohe Integrationswahrscheinlichkeit implizieren. Deshalb kommt es bei dieser Zielgruppe nur nachgeordnet auf berufspezifische Kenntnisse oder ein Arbeitsplatzangebot an. Die Punktevergabe im Rahmen dieser Säule gewichtet allgemeines Humankapital und eine akademische Ausbildung vergleichsweise hoch.

Die zweite Säule steuert die Zuwanderung qualifizierter Fachkräfte, die unterhalb der akademischen Qualifikation rangierende Ausbildungsabschlüsse und Fähigkeiten mitbringen, für die ein mittel- bis langfristiger Engpass zu erwarten ist. Die Akzentuierung der Punktevergabe weist deutliche Unterschiede zur ersten Säule auf: So erhalten etwa berufsspezifische Qualifikationen in gefragten Berufsfeldern ein relativ hohes Gewicht.

Der Vorschlag sieht ferner vor, dass sich die Genehmigung der Zuwanderung im Rahmen dieser ersten beiden Säulen nach Maßgabe einer regelmäßig vorzugebenden Höchstquote vollzieht. Innerhalb der Quotierung kommen die besten Bewerbungen zum Zug, wobei nur oberhalb eines zweiten, hoch angesetzten Schwellenwerts ein sofortiges Daueraufenthaltsrecht vergeben wird.

Die dritte Säule des vorgeschlagenen Systems ermöglicht temporäre Zuwanderung zur Deckung eines kurzfristigen Zuwanderungsbedarfes aufgrund berufsspezifischer Nachfrageüberschüsse nach Arbeitskräften. Im Rahmen dieser Säule ist die Zuwanderung strikt befristet. Die Arbeitserlaubnis ist an ein Arbeitsplatzangebot gebunden und wird zunächst für die Dauer des Arbeitsvertrages gewährt, jedoch maximal für 3 Jahre. Im Verlauf dieser Zeit 
ist eine Bewerbung im Punkteverfahren möglich. Es zudem vorgesehen, von den Unternehmen, die eine offene Stelle mit einem temporären Zuwanderer dieser Kategorie besetzen möchten, eine Gebühr zu erheben.

Der Vorschlag sieht für die ersten beiden Säulen (Hochqualifizierte und qualifizierte Fachkräfte) in einer zweijährigen Phase eine Gesamtquote von 100.000 Personen vor (jeweils 50.000 Personen pro Säule), wobei eine sukzessive Erhöhung dieser Quoten im weiteren Verlauf möglich ist. Die dritte Säule (einfach qualifizierter Zuwanderer in Berufen mit kurzfristigen Engpässen) könnte zunächst ein knappes Kontingent von 10.000 bis 20.000 temporären Zuwanderern in einer Erprobungsphase beinhalten.

Durch eine regelmäßige Überprüfung des Punktesystems und der Punktevergabe sowie der Quoten soll die nötige Flexibilität und Anpassungsfähigkeit des Regelwerks gewährleistet werden.

\section{Die „Rot-Weiß-Rote Karte“ in Österreich}

Die Zuwanderungspolitik Österreichs weist in mancher Hinsicht Parallelen zur deutschen Politik auf. In beiden Ländern wurde lange Zeit ein Zuwanderungsbedarf negiert, und der Erfolg von Kurskorrekturen in der jüngeren Zeit leidet unter anderem an der mangelhaften Wahrnehmung als potenzielle Zielländer für hoch qualifizierter Zuwanderer. Im Gegensatz zu Deutschland hat Österreich jedoch einen Kurswechsel vollzogen und mit der „Rot-Weiß-Roten Karte“ eine ökonomische Komponente in der österreichischen Zuwanderungsgesetzgebung vorgelegt.

Die „Rot-Weiß-Rote Karte“ betrifft ausschließlich Angehörige von Drittstaaten. Es erfolgt eine Differenzierung zwischen besonders hoch qualifizierten Spezialisten, Wissenschaftlern und Führungskräften sowie anderen, zur Ausübung von Mangelberufen qualifizierten Bewerbern. Die Identifikation von Mangelberufen erfolgt anhand einer jährlichen 
Mangelberufsverordnung und erscheint vergleichsweise unbürokratisch. Gleichzeitig wird auch sonstigen „Schlüsselkräften“ die Einreise gestattet, wenn eine Vorrangprüfung ergibt, dass für die angestrebte Tätigkeit kein bevorrechtigter Inländer zur Verfügung steht. ${ }^{16}$

In allen Fällen wird die „Rot-Weiß-Rote Karte“ befristet erteilt und gilt arbeitsplatzgebunden zunächst nur für ein Jahr. Sie kann aber schrittweise bis auf fünf Jahre verlängert werden. Gelingt im ersten Aufenthaltsjahr eine zehnmonatige qualifikationsgerechte Erwerbstätigkeit, wird die Karte in eine „Rot-Weiß-Rote Karte Plus“ umgewandelt. Diese entkoppelt das Aufenthaltsrecht vom bestehenden Arbeitsplatz und ermöglicht einen dauerhaften, unbeschränkten Arbeitsmarktzugang. Als Grundlage für die Vergabe der „Rot-Weiß-Roten Karte“ dient ein Punktesystem, welches das Alter, die Qualifikation, die Berufserfahrung und die Sprachkenntnisse der Bewerbenden berücksichtigt.

Durch die „Rot-Weiß-Rote Karte“ wurde zudem eine deutlich familienfreundlichere Option einer sofortigen Miteinreise von Familienangehörigen geschaffen. Für ausländische Studienabsolventen besteht schließlich die Möglichkeit, nach ihrem Studienabschluss weitere sechs Monate nach einem Arbeitsplatz zu suchen.

Das österreichische Modell erscheint insgesamt gelungen und in weiten Teilen auch auf Deutschland übertragbar. Auffällig - und aus ökonomischer Sicht richtig - ist die eher schwache Gewichtung von Sprachkenntnissen, bei denen auch Englischkenntnisse ins Feld geführt werden können. Dies erscheint für die Zielgruppe, tendenziell im internationalen Umfeld englischsprachig tätige Führungskräfte, sehr sinnvoll.

\footnotetext{
${ }^{16}$ Erfahrungsgemäß schrecken allerdings Vorrangprüfungen wegen ihrer Intransparenz die Unternehmen vor einem Zuwanderungsengagement ab.
} 
Zum jetzigen Zeitpunkt bleibt allerdings abzuwarten, inwieweit sich Österreich mit diesem Konzept im weltweiten Wettbewerb um qualifizierte Fachkräfte mittelfristig erfolgreich etablieren kann.

\section{Fazit und Ausblick}

Arbeitskräftemobilität verbessert die Allokation und die Verteilung der volkswirtschaftlichen Ressourcen. Angesichts des Fachkräftemangels bei unverändert hoher Arbeitslosigkeit unter gering Qualifizierten bleibt die Anwerbung internationaler Fachkräfte eine zentrale Aufgabe. Mit der europäischen Integration sind zudem Wechselkurse und Außenwirtschaftspolitik als Flexibilitätsinstrumente entfallen - auch deswegen hat sich die Bedeutung flexibler Arbeitsmärkte verstärkt. Initiativen zur Stärkung der ökonomischen Komponente von Zuwanderung sind deshalb von erheblicher Bedeutung.

Deutschland und Österreich sollten allerdings nicht mit $\mathrm{zu}$ hohen Erwartungen an eine Reform der Zuwanderungspolitik herangehen. Viele Wanderungswillige haben sich bereits für andere Zielländer innerhalb und außerhalb Europas entschieden. Beide Staaten werden energisch in einem weltweiten Wettbewerb um Fachkräfte werben müssen, um sich als Zielland für die international gesuchten Spitzenkräfte zu etablieren. 